\title{
Estimativa da evapotranspiração na Bacia Amazônica (*)
}

\author{
N. A. Villa Nova (') \\ E. Salati (2) \\ Ex Matsui ( ${ }^{3}$ )
}

\section{Resumo}

O balanço hídrico da Bacia Amazônica é um problema de difícil solução, não somente pelas dimensões e características da região, como também pela escassez de dados meteorológicos e hidrologicos. Procurando estimar a grandeza dos principais componentes do balanço hídrico, foi realizado um estudo com dados da Região Amazônica brasileira e de algumas estações de outros países. Fez-se o balanço de energia e, com base no mesmo, fez-se o balanço hídrico da região, adaptando-se o método de Penman para as condições de florestas. Os dados obtidos indicam que $90 \%$ da evapotranspiração deve-se ao balanço de energia. A evapotranspiração real, deve, nesta região, ser aproximadamente igual à potencial e a média encontrada foi da ordem de $4 \mathrm{~mm} /$ dia, ou seja, $1460 \mathrm{~mm}$ ano. Em uma primeira aproximação, a Bacia Amazônica funciona como ym sistema que recebe, através da precipitação, 1 年 $\times 10^{12} \mathrm{~m}^{3}$ de água por ano, sendo este total balanceado por uma descarga superficial

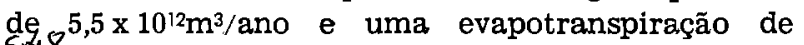
\& $10^{12} \mathrm{~m}^{3} /$ ano. Desde que a transpiracão pelas plantas representa $4 \%$ da indica que um desmatamento intensivo deverá tra. zer alterações no ciclo hidrológico.

\section{INTRODUÇÃo}

O balanço hídrico da Bacia Amazônica é um problema de difícil solução, não só pelas dimensões e características da região, mas especialmente pela escassez de dados meteorológicos $\epsilon$ hidrológicos. A descarga do rio Amazonas em óbidos foi determinada de maneira direta recentemente Oltman (1964 e 1966). A descarga dos afluentes é ainda desconhecida na sua maioria, sendo que algumas medidas de vazões relativas dos rios Negro e Solimões, foram feitas por processos indiretos através das concentrações de ${ }^{18} \mathrm{O}$ das águas daqueles rios, Matsui et al. (1973).
Pelos dados de Oltman et al (1966), a descarga média do rio Amazonas em Óbidos, é de $157.000 \mathrm{~m}^{3} \mathrm{~s}^{-1}$ com uma variação de 85.000 $\mathrm{m}^{3} \mathrm{~s}^{-1}$ (em novembro) a $240.000 \mathrm{~m}^{3} \mathrm{~s}^{-1}$ (em maio e junho). A área drenada até óbidos é cerca de $5.000 .000 \mathrm{~km}^{2}$ e a área total da bacia até a foz é aproximadamente $6.000 .000 \mathrm{~km}^{2}$. Com base em algumas medidas feitas no rio Tocantins e em dados de precipitação Oltman estimou que a descarga média do rio Amazonas na foz, é da ordem de $175.000 \mathrm{~m}^{3} / \mathrm{s}$. Pelos dados de precipitação apresentados pelo Departamento Nacional de Meteorologia e pelos avalores de estações meteorológicas da Colômbia, a precipitação média da bacia deverá ser da ordem de $2.000 \mathrm{~mm}$ por ano.

Assim, a precipitação média total na $\mathrm{Ba}$ cia Amazônica é da ordem de $12,0 \times 10^{12} \mathrm{~m}^{3}$ por ano, enquanto que a vazão na foz é da ordem de $5,52 \times 10^{12} \mathrm{~m}^{3}$ por ano, ou seja, a quantidade de água precipitada é aproximadamente 2,2 vezes maior que a água que se esçoa pela caIha principal do Amazonas, voltando ao oceano. Estes dados, embora escassos e de precisão limitada, indicam que a evaporação e a evapotranspiração devem ser responsáveis por uma parte relativamente ponderável do balanço hídrico da região. Por outro lado, as medidas de interação entre a Bacia Amazônica, as Bacias do Orenoco e a Platina são bem pouco conhecidas, embora saiba-se que existem áreas de interligação entre as mesmas.

Enquanto outras pesquisas estão sendo desenvolvidas por métodos isotópicos, (Salati et al . 1973), a fim de se estimar a recirculaçăo interna de vapor d'água, o presente trabalho tem por finalidade estabelecer, com base em alguns dados disponiveis, uma estimativa da evapotranspiração para a floresta Ámazônica.

\footnotetext{
(") - Trabalho realizado com o auxílio da CNEN e da FAPESP (Processo-Física 73/479) e coordenado pelo DPCT da CNEN.

(1) - Escola Superior de Agricultura "Luiz de Queiroz" (ESALQ), Piracicaba, SP.

( 2 ) - Centro de Energia Nuclear na Agricultura (CENA) e Escola Superior de Agricultura "Luiz de Queiroz" (ESALQ), Piracicaba, SP.

(3) - Centro de Energia Nuclear na Agricultura e Centro Nacional de Energia Nuclear.
} 
Foi adotado nesta primeira estimativa, o método de Penman (1948), que combina as equações de balanço de energia com as de transferência de massa. A adaptação deste método para as supertícies cobertas por florestas foi feita seguindo as técnicas propostas por Shiau et al. (1973).

\section{Material e mÉtodo}

\section{O MÉTODO DE PENMAN}

O método de Penman (1948) para cálcuio de evapotranspiração é expresso pelo conjunto das equações:

$$
\begin{aligned}
& E_{T}=E_{H}+E_{A} \ldots \ldots \ldots \ldots \text { (1) onde, } \\
& E_{H}=\frac{S / Y}{S / Y+1} H \ldots \ldots \ldots \\
& E_{A}=\frac{1}{S / Y+1} E_{a} \ldots \ldots \ldots \text { (3), onde, } \\
& H=\underset{0}{Q}\left(a+\frac{n}{N}\right)(1-R)-O T\left(a^{\prime}-b^{\prime} V^{-}\right) \\
& \left(a "+b " \frac{n}{N}\right) \ldots \ldots \ldots \text { (4) , } \\
& E_{a}=0.35\left(1+0,54 V_{2}\right)(e s-e)(5), \\
& \text { cujos termos significam, } \\
& E_{T}=\text { evapotranspiração potencial }(\mathrm{mm} / \mathrm{dia}) \text {. } \\
& E \text { fração de } E \text { devida ao balariço de } \\
& \text { energia }(\mathrm{mm} / \mathrm{dia}) \text {. } \\
& E_{A}=\text { fração de } E_{T} \text { devida ao déficit de sa- } \\
& \text { turação do ar. } \\
& H \text { = balanço de energia local }(\mathrm{mm} / \mathrm{dia}) \text {. } \\
& \mathrm{E} \text { = poder evaporante do ar à sombra } \\
& \text { a } \\
& \text { (mm/dia) . } \\
& \frac{S}{Y}=\text { uma constante função da temperatu } \\
& \text { ra média do ar. }
\end{aligned}
$$

$$
\underset{0}{0}=\text { constante solar diária }(\mathrm{mm} / \mathrm{dia}) \text {. }
$$

a e b = parâmetros de transparência da atmosfera para onda curta.

$a^{\prime}$ e $b^{\prime}=$ parâmetros de transparência da atmosfera para onda longa.

$$
\begin{aligned}
\mathrm{R}= & \underset{\text { poder refletor da superfície evapo. }}{\text { rante. }} \\
\mathrm{o}= & \underset{2,01 \times 10^{-9} \mathrm{~mm} \mathrm{H}_{2} \mathrm{O} / \text { dia }^{\circ} \mathrm{K}^{4}}{2,0 n s t a n t e} \text { de Stefan-Boltzmann }= \\
\frac{\mathrm{n}}{\mathrm{N}}= & \text { razão de insolação no período con- } \\
& \text { siderado. }
\end{aligned}
$$
0 constante de Stefan-Boltzmann = $2,01 \times 10^{-9} \mathrm{~mm} \mathrm{H}_{2} \mathrm{O} / \mathrm{dia}^{\circ} \mathrm{K}^{4}$
$\frac{\mathrm{n}}{\mathrm{N}}=$ razão de insolação no período con-
$\mathrm{N}$

$\mathbf{T}=$ temperatura média do ar à sombra $\left({ }^{\circ} \mathrm{K}\right)$.

e $=$ tensão parcial de vapor $(\mathrm{mm} \mathrm{Hg})$.

$\mathrm{e}=$ tensão de saturação de vapor d'água s a temperatura do ar $(\mathrm{mm} \mathrm{Hg})$.

$\mathrm{V}=$ velocidade do vento, medida a $2,0 \mathrm{~m}$ 2 acima da superfície evaporante $(\mathrm{m} / \mathrm{s})$.

O conjunto de equações (1), (2), (3), (4) e (5) tem sua aplicação restrita à cobertura vegetal de porte médio, quando se observam condições para ocorrência da taxa de evapotranspiração potencial, - sendo os dados de $\mathrm{n} / \mathrm{N}, \mathrm{T}_{\mathrm{a}}$, e e $\mathrm{V}_{2}$ coletados em estação climatológica comum.

ADAPTAÇÃO DO MÉTODO DE PENMAN

PARA COBERTURA VEGETAL DE GRANDE PORTE

\section{Transformação de dados observados na superfície}

As modificações realizadas são aquelas sugeridas por Shiau et al. (1973), relativas à transformação dos dados observados em estação climatológica padrão, para dados da parte superior de um maciço florestal de mesma latitude e altitude, como se segue:

Velocidade do vento ao nível da copa 
Em estações climatológicas padrão medese usualmente a velocidade de fluxo livre do vento, geralmente a $10 \mathrm{~m}$ de altura $\left(\mathrm{V}_{10}\right)$. De acordo com Geiger (1966) a velocidade média do vento no interior da parte superior da copa $\left(V_{c}\right)$ é cerca de $40 \%$ da velocidade de fluxo livre $\left(V_{10}\right)$ ou seja:

$$
v_{2} \simeq v_{c} \simeq 0,4 V_{10} \ldots \ldots \ldots \ldots .
$$

Desde que, devido à própria distribuição de radiação e vento, a parte superior da copa é a zona mais ativa no processo evaporativo, a expressão (5) modificada para cobertura vegetal de grande porte tornar-se-á:

$$
\underset{\mathrm{m}}{\mathrm{a}_{\mathrm{m}}}=0,35\left(1+0,54 \mathrm{~V}_{\mathrm{c}}\right)\left(\mathrm{e}_{\mathrm{s}}-\mathrm{e}\right) \ldots
$$

ou ainda, de acordo com (6),

$$
\begin{aligned}
& E=0,35\left(1+0,216 V_{10}\right)\left(e_{8}-e_{c}\right)(8) \text {, } \\
& \text { a } \\
& \text { n } \\
& \text { onde, } \\
& \mathrm{E}=\text { poder evaporante do ar na parte } \\
& \text { a } \\
& \mathrm{m} \\
& \text { superior do maciço }(\mathrm{mm} / \mathrm{dia}) \text {. } \\
& V_{10}=\text { velocidade de fluxo livre do vento } \\
& (\mathrm{m} / \mathrm{s}) \text {. } \\
& \mathrm{e}_{\mathrm{s}}=\text { tensão de saturação à temperatura } \\
& \text { do ar. } \\
& e_{c}=\text { tensão parcial de vapor na parte } \\
& \text { superior da copa. }
\end{aligned}
$$

Pressâo parcial de vapor ao nível da copa

A pressão parcial da atmosfera em estações climatológicas é derivada da umidade relativa pela equação:

$$
e=0,01 \text { (U.R.) } e_{s} \ldots \ldots \ldots \ldots \ldots
$$

Geiger (1966) demonstra que são peque nas as diferenças de pressão de vapor entre o nível do solo e a copa, em uma floresta, desde que existe um fluxo ascendente de vapor d'água. Para o caso de extensas regiões de floresta tropical, não devemos esperar grandes diferenças entre a pressão de vapor medida $\mathrm{Em}$ estação climatológica situada em local aberto, e o nível da copa das árvores, de tal modo que com boa aproximação poderemos considerar:

$$
\mathrm{e}_{\mathrm{c}} \simeq \mathrm{e}
$$

\section{Adaptação de parâmetros}

\section{Parâmetros $a$ e $b$}

De acordo com Glover \& McCulloch (1958), o valor dos parâmetros $a$ e $b$ não são universais devendo ser determinados para cada re. gião. Citamos abaixo algumas determinações realizadas:

a) Virgina, USA: $a=0,22, b=0,54$, Kimball (1914) .

b) Canberra, Austrália: $a=0,25, b=0,54$, Prescott (1940).

c) Rothamsted, Inglaterra: $a=0,18, b=$ 0,55, Penman (1948).

d) São Paulo, Brasil (média do estado): $a=0,24, b=0,58$, Cervellini et al. (1966)

e) Piracicaba, Brasil: $a=0,26, b=0,51$, Ometto (1967).

f) Petit-Bourg, Guadeloupe: $a=0,26, b=$ 0,51 , Bonhome et al., (1973).

Em face de tais dados adotamos os valores $a=0,26$ e $b=0,51$, determinados por Ometto e Bonhome em condições tropicais úmidas .

Parâmetros a', b' e a", b"

No equacionamento de Penman os valores adctados de a'b' e a“'b", pelos bons resultados obtidos, são os seguintes:

$$
\begin{array}{ll}
a^{\prime}=0,56 & b^{\prime}=0,09 \\
a^{\prime \prime}=0,10 & b^{\prime \prime}=0,90
\end{array}
$$

sendo $a^{\prime}$ e $b^{\prime}$ dependentes da tensão parcial de vapor (e) e a", b" função do tipo e altura de nuvens. 
Segundo Shiau (1973), para o caso de maciços florestais, o erro decorrente do uso de tais valores é bastante atenuado se considerarmos períodos mensais de estimativa. $\mathrm{Na}$ falta de avaliaçốes de tais parâmetros para nossas condições, utilizaremos esses mesmos valores.

\section{Poder refletor}

De acordo com Reifsnyder et al. (1965), o albedo de maciços de coníferas oscila entre 0,11 e 0,20 ao longo das estações do ano. Para condições de mata tropical sempre verde, julgamos coerente o uso da média destes valores, ou seja: $R=0,15$.

\section{A equação modificada de Penman}

Em face ao conjunto de considerações expostas, a equação de Penman, adaptada para vegetação tropical de grande porte e extensão, tornar-se-á:

$$
\begin{aligned}
& \mathrm{T}_{\mathrm{m}}=\mathrm{H}_{\mathrm{m}}+\underset{\mathrm{m}}{\mathrm{A}_{\mathrm{m}}} \ldots \ldots \ldots \text { (11) onde, } \\
& E_{\mathrm{m}}=\frac{S / Y}{S / Y+1} \cdot{ }_{m} \ldots \ldots \ldots \ldots(12) . \\
& E_{m}=\frac{1}{S / Y+1} \cdot E_{\mathrm{m}} \ldots \ldots \text { (13) onde, } \\
& H_{m}=Q \underset{o}{\left(0,26+0,51 \frac{n}{N}\right)}(1-R)-O T_{a}^{4} \\
& (0,56-0,09 \sqrt{ } \mathrm{e})\left(0,1+0,9 \frac{\mathrm{n}}{\mathrm{N}}\right)(14) \text {, } \\
& \underset{\mathrm{m}}{\mathrm{E}_{\mathrm{m}}}=0,35(1+0,216 \mathrm{~V} \underset{10}{)}(\mathrm{e}-\mathrm{s})(15)
\end{aligned}
$$

onde os índices $m$ se referem a "maciço florestal" e $V_{\text {to }}$ a velocidade de fluxo livre de vento, geralmente medida a $10,0 \mathrm{~m}$ em estação climatológica padrão; os outros símbolos têm o significado original.

\section{RESULTADOS OBTIDOS E DISCUSSÃO}

Os dados, de temperatura média, insolação, umidade relativa, precipitação e velocidade de vento, para as cidades estudadas estão indicados na Tabela 1.

Pelo método exposto no parágrafo 2, foram calculados $E$ e $E$, para as cidades $m \quad m$

de Belém, Manaus e Benjamin Constant, que estão aproximadamente na mesma iatitude ao longo do rio Amazonas. Nestes locais as mé. dias diárias foram calculadas a partir de 24 medidas horárias. Os dados obtidos estão indicados nas figuras 1, 2 e 3 , nos quais são indicados ainda os valores de $\mathrm{Q}$ e $\mathrm{Q}$, sendo $Q_{s}=Q_{0}\left(a+b \frac{n}{N}\right)$, a estimativa da energia solar que atinge a superfície. Os dados de energia estão expressos em milimetros equivalentes de água evaporada $\{1 \mathrm{~mm}$ de água corresponde a $59 \mathrm{call}$. Note-se que os valores do balanço de energia encontrados concordam em ordem de grandeza com os determinados por Costa Azevedo et al. (1974).

Na Tabela II, estão os valores mensais de $\mathrm{H}_{\mathrm{m}}, \mathrm{T}_{\mathrm{m}}$ e a relaçăo $\mathrm{f}=\mathrm{H}_{\mathrm{m}}^{\mathrm{E}} \underset{\mathrm{m}}{\mathrm{T}}$ para cada mês nas cidades de Belém, Manaus e Benjamin Constant. Os valores de $f$ indicados nesra Tabela constituem a fração corresponciente à contribuição do balanço de energia na evapotranspiração potencial; a fração da contribulção do "fator aerodinâmico" pode ser obtida pela relação $(1-f)$. Na Tabela II, estão também indicados os valores médios de $f$, para as 3 cidades. De maneira geral encontrou-se que cerca de $90 \%$ da evapotranspiração potencial, é devida ao balanço de energia, enquanto que cerca de $10 \%$ é devida ao "fator aerodinâmico" dependente da umidade relativa do ar, da temperatura e da velocidade do vento. $\mathrm{Na}$ Tabela III são relatados os valores médios mensais de evapotranspiração potencial, estimados pelo método proposto, para as diferentes localidades estudadas. 


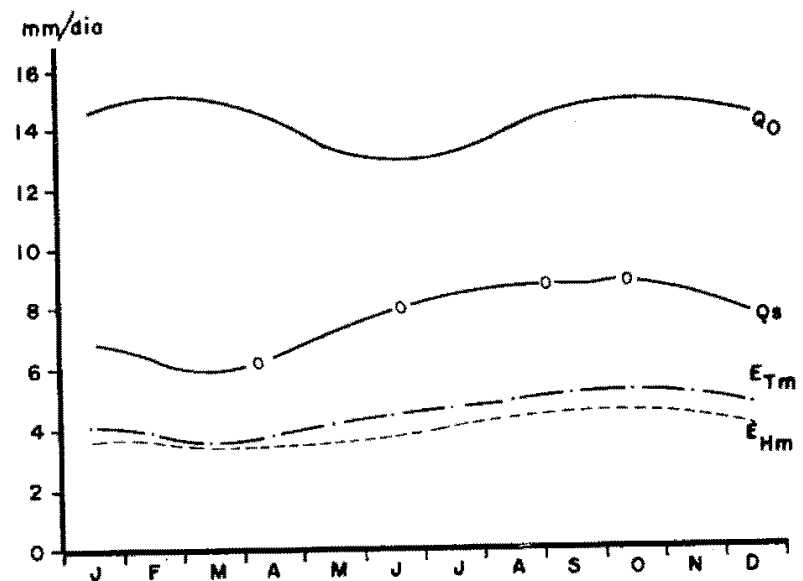

Fig. 1 - Valor médio mensal dos componentes do balanço de energia $\left(Q_{0} e Q_{s}\right)$ e evapotranspiração potencial ( $\mathbf{E}_{\text {Tm }}$ e $\mathbf{E}_{\mathrm{Hm}}$ ) da estação de Belém(PA).

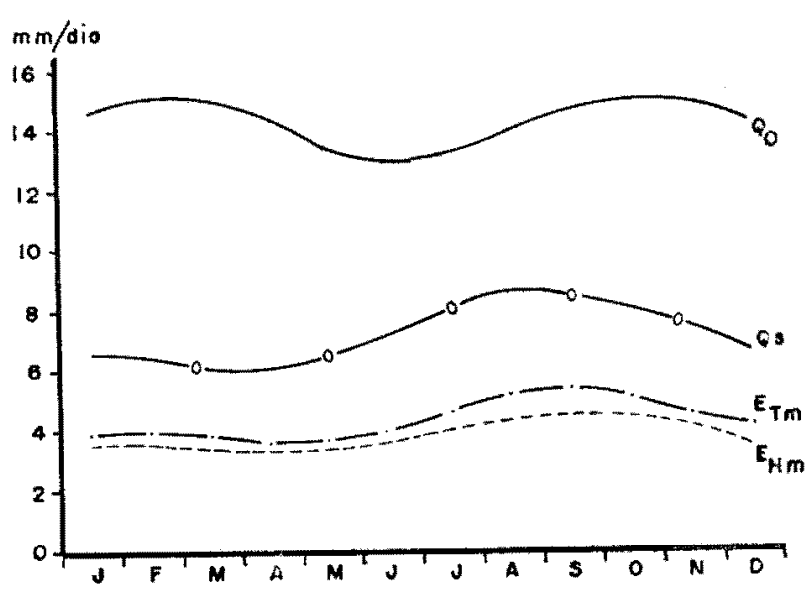

Fig. 2 - Valor médio mensal dos componentes do balanço de energia $\left(Q_{0}\right.$ e $\left.Q_{s}\right)$ e evapotranspiração potencial (ETm e E Hm) da estação de Manaus (AM) .

Os valores médios ponderados de acordo com a distribuição dos pontos de observação, indicam que a evapotranspiração potencial média para a área da Bacia Amazônica é da ordem de $4 \mathrm{~mm}$ por dia, ou seja $1.460 \mathrm{~mm}$ por ano. Pelo aspecto da floresta [sempre verde e em constante crescimento vegetativo de reposição), nas partes baixas da bacia, pode-se admitir que a evapotranspiração potencial seja próxima da evapotranspiração real. Sendo a precipitação média, $2.000 \mathrm{~mm}$ por ano, vê-se

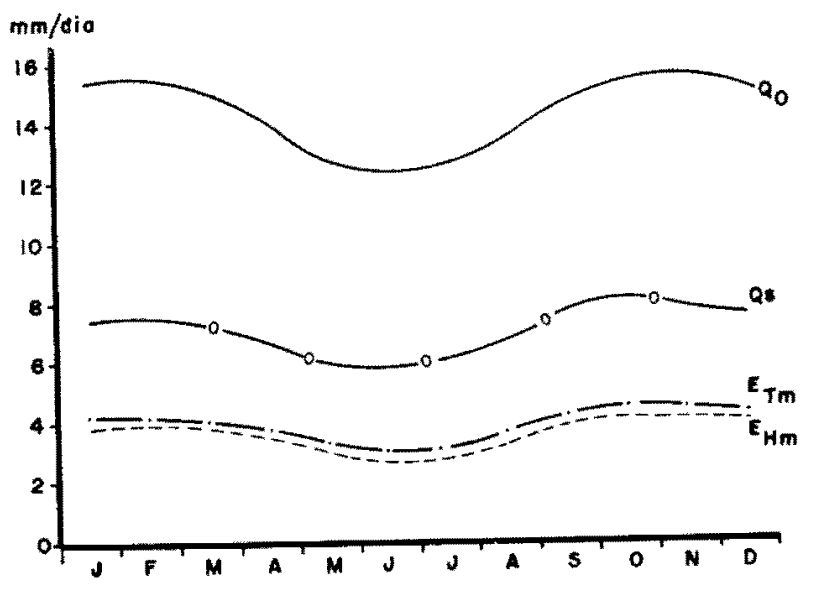

Fig. 3 - Valor médio mensal dos componentes do balanço de energia $\left(Q_{0}\right.$ e $\left.Q_{S}\right)$ e evapotranspiração potencial (ETm e EHm) da estaçăo de Benjamin Constant(AM).

que a evapotranspiração potencial correspon. ue a $73 \%$ da precipitação. Nas paisagens bo tânicas denominadas "cerrados", a situação pode ser diferente, porque a evapotranspiração real é menor do que a potencial.

Numa primeira aproximação, a Bacia Ama. zônica, aproxima-se de um sistema que recebe por precipitação $12 \times 10^{12} \mathrm{~m}^{3}$ de água po: ano, sendo este total balanceado por uma descarga superficial de $5,52 \times 10^{12} \mathrm{~m}^{3}$ por ano e uma evapotranspiração real estimada de $6,48 \times$ $10^{12} \mathrm{~m}^{3}$ por ano.

Como vimos, o balanço de energia é respcnsável em aproximadamente $90 \%$ de evapotranspiração potencial. Sabe-se que qualquer modificação nas caracteristicas da copertura uo solo implicaria não só em alterações nos parâmetros do balanço de energia, como tam. bém na dinâmica do escoamento, afetando diretamente o processo evaporativo. Como consequência, uma provável diminuição da umidade relativa deverá determinar, a exemplo dos climas desérticos, maiores oscilaçōes térmicas, e profundas alterações no equilíbrio dos sistemas convectivos característicos da regiăo.

Pretendem os autores deste trabalho, numa fase posterior, anexando um maior número de dados, complementá-lo, com o objetivo de aprimorar as estimativas realizadas. 


\section{Agradecimentos}

Os autores agradecem o Diretor e os funcionários do Departamento Nacional de Meteorologia do M. A. e o capitão C. Tatagiba do C. T. A pelo auxílio na obtenção de alguns dados meteorológicos.

\section{SUMMARY}

The establishment of a water balance for the Amazon Basin constitutes a problem of difficult solution, not only on the account of its extension and characteristics, but also for lack of sufficient meteorological and hydrological data. In an attempt to estimate the magnitude of the main components of the water balance, a study was made with data from the Brazilian Amazon Region and from some observation stations in other countries.

An energy balance was made and based on this balance the water balance of the region was established, having the Penman method been adapted to forest conditions. The data obtained indicate that $90 \%$ of the evapotranspiration is due to the energy balance. The evapotranspiration in this area should be very close to the potential evapotranspiration, and the average found was of the order of $4 \mathrm{~mm} /$ day, i.e., $1460 \mathrm{~mm} /$ year. As a first approximation it was foynd that the Amazon Basin system receives $1 \frac{1}{4}, 4 \times 10^{12} \mathrm{~m}^{3}$ waíer/year through precipitation, this total being balanced by a surface discharge of $5.5 \times 10^{12} \mathrm{~m}^{3} /$ year and an evapotranspiration of $10^{12} \mathrm{~m}^{3} /$ year Since transpiration by plants represents $4 \%$ of the all seems to indicate that intensive deforestation shall bring about alterations of the hydrologic cycle.

\section{BIBLIOGRAFIA CITADA}

Bonhome, R. \& GRANCHER, C.V.

1974 - Measurement e estimation of evapotranspiration in French West Indies. In: Annual meeting of the Caribbean Food Crops Society, 11., Barbados.

Cerveluint, a.; Salati, E. \& Godox, H.

1966 - A estimativa da distribuição da ener. gia solar no Estado de São Paulo. Bragantia, $25: 31-40$.

Costa Azevedo, D.; Pinto M. M. \& Azevedo M.L.

1974 - Distribuição da radiação solar na região amazônica. In: Simpósio Brasileiro de Energia Solar, 2., João Pessoa.

GEIGER, $R$.

1966 - The climate near the ground. Mass., Harvard University Press.
GLOVER, J. \& MCCULLOGH, J.S.G.

1958 - The empirical relation between solar radiation and hours of sunshine. Qua terly Journal of the Royal Meteorological Society, 84: 172 .

KIMB

1914 - Montly Weather Review, 42:474. Apud Shiau, S.Y. \& Davar, K.S.

Matsui, E.; Salati, E.; Brinkmann, W.L.F.

\& FRIEDMAN, I.

1972 - Vazões relativas dos rios Negro e Solimões através das concentrações de 18O. Acta Amazonica, 2(3):31-37.

OLtMan, R.E.

1967 - Reconnaissance investigations of the discharge and water quality Amazon. In: Atas do Simpósio sobre a Biota Amazonica, 3 (Limnologia) : 163-185.

Oltman, R.E.; Sternderg. H.O.R.;

AMEs, F.C. \& DAvis JR., L.C.

1964 - Amazon river investigations reconnaissance measurements of July 1963. Geological Survey Circular, 486.

OMETrO, J.C.

1967 - Estudo das relações entre Radiação Solar Global, Radiação Líquida e Insolação. Piracicaba, SP. (Tese de doutoramento-ESALQ)

Penman, H.L.

1948 - Natural evaporation from open water, bare soil and grass. Proc. R. Soc. Ser. A, London, 193: 120-145.

PRESCOTT, J.A.

1940 - Trans. R. Soc. South Australia $64: 114$. Apud Shiau, S.V. \& Davar, K.S.

REFSNEIDER, W.E. \& LULL, H.V.

1965 - Radiant energy in relation to forest. U.S. Dep. Agric. Forest Service, Tech. Bull., 1344.

Salati, E.; Matsui, E.; Cervellini, A.

\& FrIEDMan, I.

1973 - Isotope Hydrology in Amazonia - I $\delta 18_{0}$ and $\delta_{D}$ in river waters. In press, 1973.

Salati, E.; Ometto, J.C.; Cerveluni, A.;

Villa Nova, N.A. \& SANTos, J.M.

1966 - Estudo de determinação da radiação solar em Piracicaba, SP. Boletim Técnica, S.M.M. Agricultura, 5:1-5.

Shiau, S.V. \& Dayar, K.S

1973 - Modified Penman method for potencial evapotranspiration from forest regions. Journal of Hydrology, $18: 349-365$.

Villa Nova, N.A.

1974 - Estudos sobre balanço de energia em cultura de arroz. Piracicaba, SP. (Tese de Livre-Docência, ESALQ) 
APENDICE

TABELA 1 - Dados meteorológicos e coordenadas geográficas das estações

\begin{tabular}{|c|c|c|c|c|c|c|c|c|c|c|c|c|c|}
\hline $\begin{array}{c}\text { MEDIAS } \\
1961-1973\end{array}$ & \multicolumn{4}{|c|}{ Estação: ALTAMIRA } & \multicolumn{3}{|c|}{ Lat. $3^{\circ} 12$ 'S } & g. $52^{\circ}$ & \multicolumn{3}{|c|}{ Alt. $75 \mathrm{~m}$} & & \\
\hline MESES & Jan. & Fev. & Mar. & Abr. & Mai. & Jun. & Jul. & Ago. & Set. & Out. & Nov. & Dez. & Ano \\
\hline Temperatura ( $\left.{ }^{\circ} \mathrm{C}\right)$ & 25,5 & 25,1 & 25,2 & 25,2 & 25,3 & 25,4 & 25,2 & 25,9 & 26,8 & 26,5 & 26,7 & 26,0 & 25,7 \\
\hline Umid. Relat. $(\%)$ & 84 & 88 & 88 & 89 & 89 & 88 & 86 & 84 & 84 & 81 & 81 & 81 & 85 \\
\hline Vento $(\mathrm{m} / \mathrm{s})$ & 1,8 & 2,2 & 1,8 & 1,6 & 1,4 & 1,5 & 1,6 & 2,1 & 2,1 & 2,3 & 2,3 & 2,2 & 1,9 \\
\hline Chuva (mm) & 223 & 241 & 344 & 260 & 204 & 95 & 81 & 22 & 22 & 33 & 46 & 130 & 1710 \\
\hline $\mathrm{n} / \mathbf{N}$ & 0,24 & 0,23 & 0,19 & 0,22 & 0,38 & 0,18 & 0,41 & 0,55 & 0,48 & 0,34 & 0,20 & 0,23 & 0,30 \\
\hline
\end{tabular}

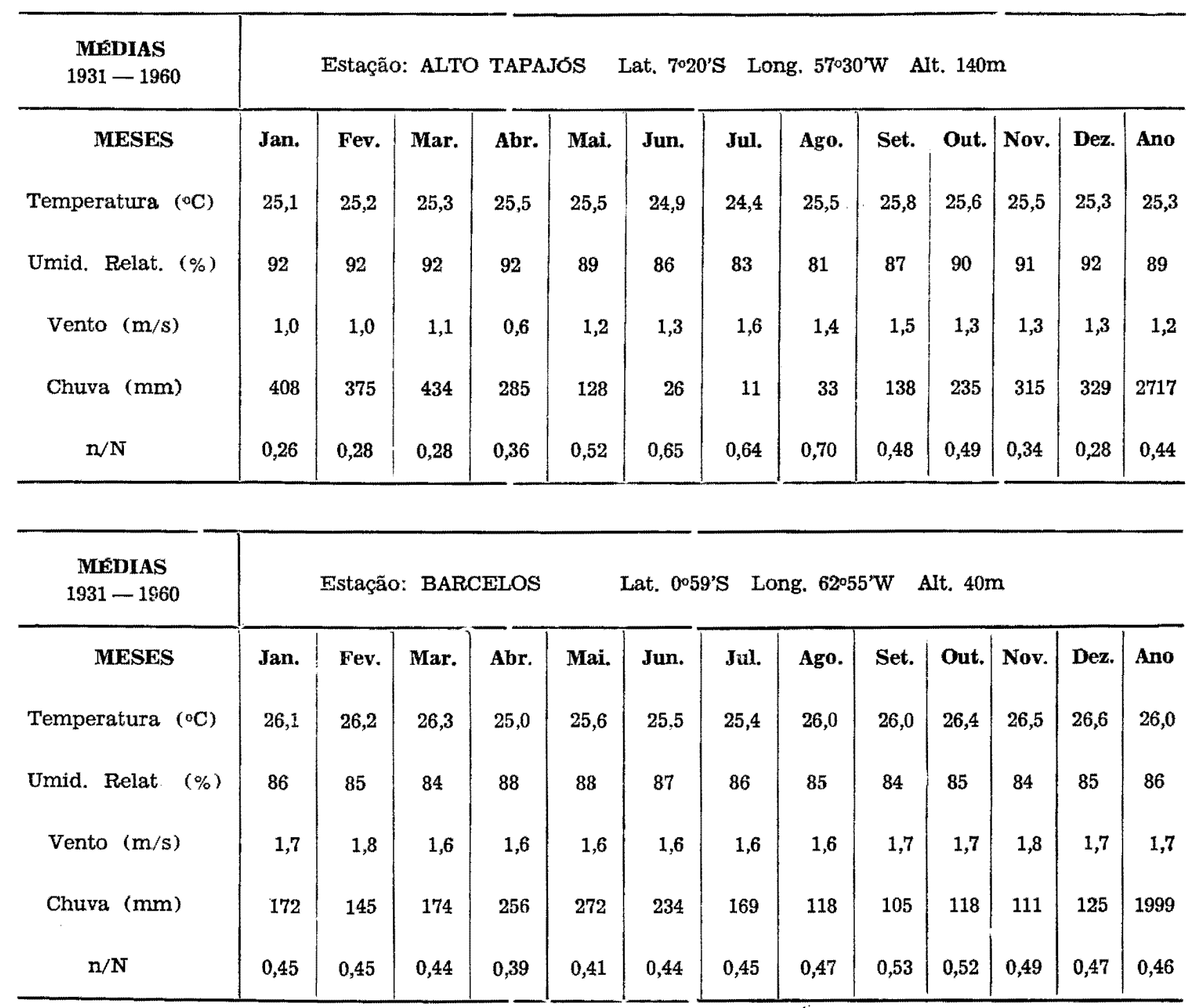


Tabela $1-$ (continuação)

\begin{tabular}{|c|c|c|c|c|c|c|c|c|c|c|c|c|c|}
\hline $\begin{array}{c}\text { MEDTAS } \\
1951-1960\end{array}$ & \multicolumn{5}{|c|}{ Estação: CONSTANT } & \multicolumn{2}{|c|}{ Lat. $4 \circ 22$ 's } & \multicolumn{2}{|c|}{ ing. $70002 \mathrm{~W}$} & \multicolumn{2}{|c|}{ Alt, $80 \mathrm{~m}$} & & \\
\hline MESES & Jan. & Fev. & Mar. & Abr. & Mai. & Jun. & JuI. & Ago. & Set. & Out. & Nov. & Dez. & Ano \\
\hline Temperatura $(\mathrm{o})$ & 24,4 & 24,3 & 24,2 & 24,2 & 24,1 & 23,4 & 22,9 & 23,8 & 24,5 & 24,6 & 24,7 & 24,6 & 24,2 \\
\hline Umid. Relat. (\%) & 89 & 90 & 91 & 90 & 90 & 86 & 87 & 88 & 88 & 89 & 90 & 91 & 89 \\
\hline Vento $(\mathrm{m} / \mathrm{s})$ & 1,5 & 1,6 & 1,5 & 1,4 & 1,4 & 1,4 & 1,3 & 1,4 & 1,4 & 1,5 & 1,5 & 1,6 & 1,5 \\
\hline Chuva (mm) & 340 & 280 & 350 & 280 & 210 & 140 & 120 & 140 & 200 & 220 & 250 & 280 & 2810 \\
\hline $\mathrm{n} / \mathrm{N}$ & 0,45 & 0,45 & 0,44 & 0,43 & 0,43 & 0,40 & 0,44 & 0,47 & 0,53 & 0,52 & 0,49 & 0,47 & 0,46 \\
\hline
\end{tabular}

\begin{tabular}{|c|c|c|c|c|c|c|c|c|c|c|c|c|c|}
\hline \multirow{2}{*}{$\begin{array}{c}\text { MEDIAS } \\
1961-1973\end{array}$} & \multicolumn{5}{|c|}{ Estação: CARAUARI } & \multicolumn{2}{|c|}{ at. $4.57 ' \mathrm{~s}$} & \multicolumn{2}{|c|}{ Long. $66^{\circ} 54^{\prime} \mathrm{W}$} & \multicolumn{2}{|c|}{ It. $81 \mathrm{~m}$} & \multirow{2}{*}{\multicolumn{2}{|c|}{ Dez: }} \\
\hline & Jan. & Fev. & Mar & Abr. & Mai & Jun. & Jul. & Ago. & Set. & Out. & Nov. & & \\
\hline Temperatura $\left({ }^{\circ} \mathrm{C}\right)$ & 25,9 & 26,2 & 25,8 & 24,9 & 25,5 & 25,3 & 24,8 & 25,2 & 25,3 & 26,1 & 25,9 & 25,8 & 25,6 \\
\hline Umid. Relat. $(\%)$ & 88 & 88 & 87 & 89 & 89 & 90 & 87 & 85 & $8 \dot{b}$ & 86 & 86 & 87 & 87 \\
\hline Vento $(\mathrm{m} / \mathrm{s})$ & 0,7 & 0,7 & 0,8 & 0,7 & 0,7 & 0,9 & 0,8 & 0,8 & 0,6 & 0,8 & 0,7 & 0,7 & 0,7 \\
\hline Chuva $(\mathrm{mm})$ & 330 & 173 & 227 & 336 & 245 & 152 & 89 & 157 & 186 & $23 u$ & 310 & 269 & 2704 \\
\hline $\mathrm{n} / \mathrm{N}$ & 0,47 & 0,32 & 0,32 & 0,29 & 0,33 & 0,46 & 0,49 & 0,50 & 0,52 & 0,43 & 0,31 & 0,27 & 0,39 \\
\hline
\end{tabular}

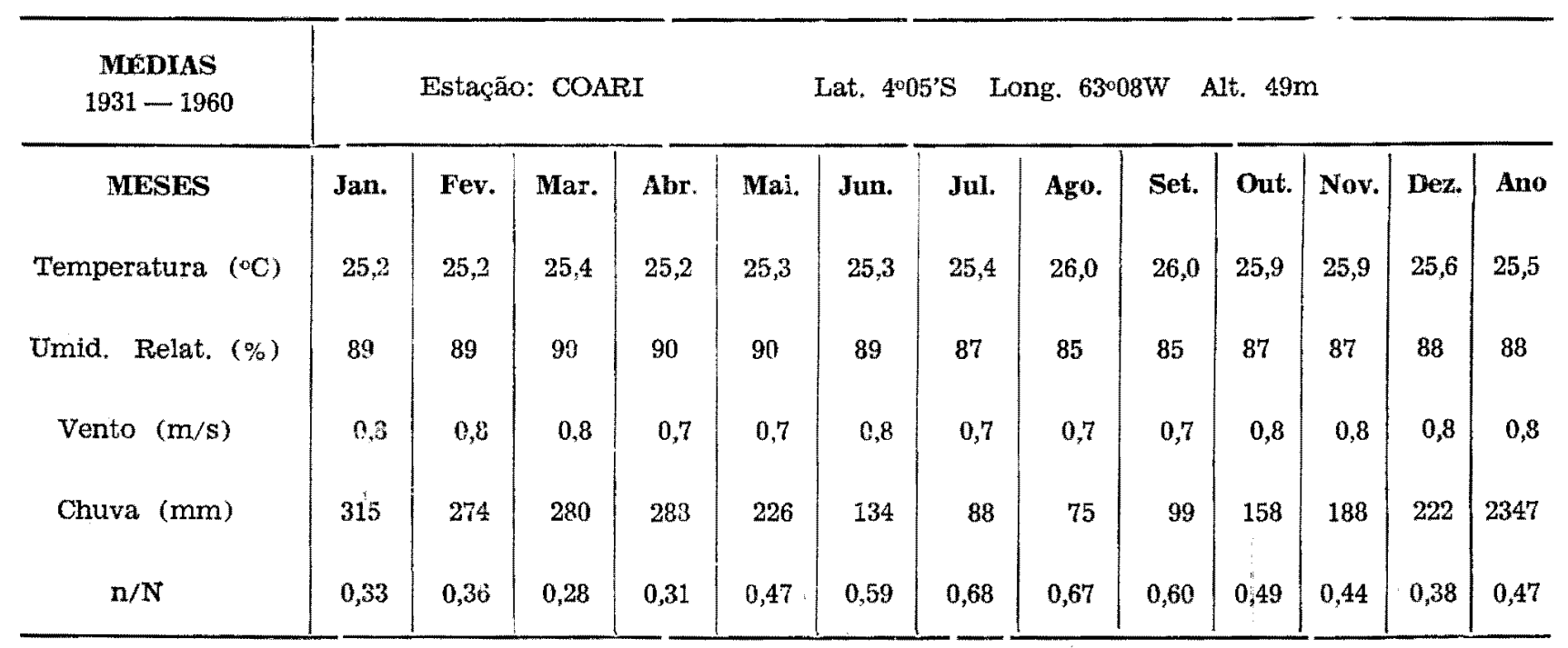


Tabela I - (continuação)

\begin{tabular}{|c|c|c|c|c|c|c|c|c|c|c|c|c|c|}
\hline $\begin{array}{c}\text { MEDIAS } \\
1931-1960\end{array}$ & \multicolumn{5}{|c|}{ Estação: FONTE BOA } & \multicolumn{2}{|c|}{ Lat. $2^{\circ} 32^{\prime} \mathrm{S}$} & \multicolumn{2}{|c|}{ Long. $66^{\circ} 10^{\prime} \mathrm{W}$} & \multicolumn{2}{|c|}{ Alt. $56 \mathrm{~m}$} & & \\
\hline MESES & Jan. & Fev. & Mar. & Abr. & Mai. & Jun. & Jul. & Ago. & Set. & Out. & Nov. & Dez. & Ano \\
\hline Temperatura $\left({ }^{\circ} \mathrm{C}\right)$ & 24,8 & 24,9 & 24,9 & 24,8 & 24,7 & 24,5 & 24,3 & 24,9 & 25,2 & 25,3 & 25,3 & 25,2 & 24,9 \\
\hline Umid. Relat. (\%) & 89 & 89 & 89 & 89 & 90 & 89 & 89 & 88 & 87 & 88 & 88 & 87 & 89 \\
\hline Vento $(\mathrm{m} / \mathrm{s})$ & 1,6 & 1,4 & 1,5 & 1,5 & 1,3 & 1,3 & 1,2 & 1,2 & 1,4 & 1,5 & 1,4 & 1,4 & 1,4 \\
\hline Chuva (mm) & 298 & 237 & 278 & 336 & 314 & 233 & 175 & 149 & 150 & 194 & 186 & 247 & 2802 \\
\hline$\pi / N$ & 0,45 & 0,45 & 0,43 & 0,43 & 0,42 & 0,44 & 0,45 & 0,47 & 0,52 & 0,50 & 0,48 & 0,49 & 0,46 \\
\hline
\end{tabular}

\begin{tabular}{|c|c|c|c|c|c|c|c|c|c|c|c|c|c|}
\hline $\begin{array}{c}\text { VIEDIAS } \\
1962-1973\end{array}$ & \multicolumn{4}{|c|}{ Estação: HUMAITÁ } & \multicolumn{3}{|c|}{ Lat. $7 \circ 31$ 's } & g. 63 & \multicolumn{3}{|c|}{$N$ Alt. $50 \mathrm{~m}$} & & \\
\hline MESES & Jan. & Fev. & Mar. & Abr. & Mai. & Jun. & Jul. & Ago. & Set. & Out. & Nov, & Dez. & Ano \\
\hline Temperatura $\left({ }^{\circ} \mathrm{C}\right)$ & 25,6 & 25,5 & 25,9 & 25,9 & 25,8 & 25,6 & 25,3 & 26,3 & 26,5 & 26,3 & 26,3 & 26,2 & 25,9 \\
\hline Umid. Relat. $(\%)$ & 87 & 88 & 89 & 88 & 87 & 83 & 80 & 79 & 81 & 82 & 84 & 86 & 85 \\
\hline Vento $(\mathrm{m} / \mathrm{s})$ & 1,9 & 2,0 & 1,6 & 1,8 & 1,8 & 1,8 & 1,9 & 1,9 & 1,9 & 2,0 & 2,2 & 2,1 & 1,9 \\
\hline Chuva $(\mathrm{mm})$ & 261 & 277 & 319 & 250 & 163 & 54 & 21 & 60 & 107 & 184 & 255 & 283 & 2234 \\
\hline $\mathrm{n} / \mathrm{N}$ & 0,34 & 0,29 & 0,36 & 0,30 & 0,41 & 0,53 & 0,57 & 0,61 & 0,50 & 0,50 & 0,39 & 0,41 & 0,45 \\
\hline
\end{tabular}

\begin{tabular}{|c|c|c|c|c|c|c|c|c|c|c|c|c|c|}
\hline $\begin{array}{c}\text { MEDIAS } \\
1931-1960\end{array}$ & \multicolumn{5}{|c|}{ Estação: YAUARETE } & Lat. $0^{\circ} 18^{\prime} \mathrm{S}$ & \multicolumn{5}{|c|}{ Long. $68^{\circ} 54 \mathrm{~W}$ Alt. $122 \mathrm{~m}$} & & \\
\hline MESES & Jan. & Fev. & Mar. & Abr. & Mai. & Jun. & Jul. & Ago. & Set. & Out. & Nov. & Dez. & Ano \\
\hline Temperatura $\left({ }^{\circ} \mathrm{C}\right)$ & 25,2 & 25,2 & 25,3 & 25,1 & 24,9 & 24,4 & 24,1 & 24,5 & 25,1 & 25,3 & 25,5 & 25,3 & 25,0 \\
\hline Umid. Relat. (\%) & 88 & 88 & 88 & 89 & 90 & 90 & 90 & 88 & 87 & 88 & 87 & 88 & 88 \\
\hline Vento $(\mathrm{m} / \mathrm{s})$ & 1,4 & 1,5 & 1,5 & 1,4 & 1,3 & 1,4 & 1,2 & 1,2 & 1,3 & 1,3 & 1,2 & 1,3 & 1,3 \\
\hline Chuva $(\mathrm{mm})$ & 259 & 246 & 295 & 363 & 389 & 356 & 350 & 278 & 266 & 237 & 227 & 237 & 3503 \\
\hline $\mathrm{n} / \mathrm{N}$ & 0,36 & 0,33 & 0,32 & 0,29 & 0,30 & 0,30 & 0,32 & 0,39 & 0,43 & 0,39 & 0,39 & 0,38 & 0,35 \\
\hline
\end{tabular}




\begin{tabular}{|c|c|c|c|c|c|c|c|c|c|c|c|c|c|}
\hline $\begin{array}{c}\text { MEDIAS } \\
1968-1973\end{array}$ & \multicolumn{4}{|c|}{ Estação: MACAPÁ } & \multicolumn{3}{|c|}{ Lat. $0^{\circ} 10^{\prime} \mathrm{N}$} & g. $51^{\circ}$ & \multicolumn{3}{|c|}{$V$ Alt. $10 \mathrm{~m}$} & & \\
\hline MESES & Jan. & Fev. & Mar & Abr. & Mai. & Jun & Jul. & Ago. & Set. & Out. & Nov. & Dez. & Ano \\
\hline Temperatura $\left({ }^{\circ} \mathrm{C}\right)$ & 26,2 & 26,0 & 25,5 & 25,8 & 26,0 & 26,2 & 26,1 & 26,6 & 27,2 & 27,8 & 27,5 & 27,0 & 26,5 \\
\hline Umid. Relat. $(\%)$ & 84 & 87 & 88 & 89 & 89 & 86 & 85 & 82 & 78 & 74 & 77 & 80 & 83 \\
\hline Vento $(\mathrm{m} / \mathrm{s})$ & 2,6 & 2,1 & 1,9 & 1,7 & 1,5 & 1,6 & 1,8 & 2,1 & 3,0 & 3,3 & 2,8 & 2,7 & 2,3 \\
\hline Chuva (mm) & 256 & 325 & 394 & 291 & 349 & 208 & 173 & 99 & 56 & 15 & 66 & 147 & 2379 \\
\hline $\mathrm{n} / \mathrm{N}$ & 0,46 & 0,40 & 0,29 & 0,31 & 0,38 & 0,54 & 0,77 & 0,75 & 0,77 & 0,78 & 0,73 & 0,57 & 0,56 \\
\hline
\end{tabular}

\begin{tabular}{|c|c|c|c|c|c|c|c|c|c|c|c|c|c|}
\hline $\begin{array}{l}\text { MÉDIAS } \\
1931-1960\end{array}$ & \multicolumn{4}{|c|}{ Estação: MANAUS } & \multicolumn{3}{|c|}{ Lat. $3^{\circ} 08^{\prime} \mathrm{S} \quad$ I } & \multicolumn{2}{|c|}{ s. $60^{\circ} 01^{\prime} \mathrm{W}$} & \multicolumn{2}{|c|}{ lit. $48 \mathrm{~m}$} & & \\
\hline MESES & Jan. & Fev. & Mar. & $\mathbf{A b r}$ & Mai. & Jun. & Jul. & Ago. & Set. & Out. & Nov. & Dez. & Ano \\
\hline Temperatura $\left({ }^{\circ} \mathrm{C}\right)$ & 25,9 & 25,8 & 25,8 & 25,8 & 26,4 & 26,6 & 26,9 & 27,5 & 27,9 & 27,7 & 27,3 & 26,7 & 26,7 \\
\hline Umid. Relat. (\%) & 88 & 88 & 88 & 88 & 86 & 83 & 80 & 77 & 77 & 79 & 82 & 85 & 83 \\
\hline Vento $(\mathrm{m} / \mathrm{s})$ & 2,6 & 2,7 & 2,7 & 2,4 & 2,9 & 2,6 & 3,0 & 2,7 & 3,1 & 2,8 & 2,7 & 2,7 & 2,7 \\
\hline Chuva (mm) & 276 & 277 & 301 & 287 & 193 & 98 & 61 & 41 & 62 & 112 & 165 & 228 & 2101 \\
\hline $\mathrm{n} / \mathrm{N}$ & 0,38 & 0,33 & 0,30 & 0,31 & 0,46 & 0,56 & 0,66 & 0,71 & 0,60 & 0,54 & 0,48 & 0,40 & 0,48 \\
\hline
\end{tabular}

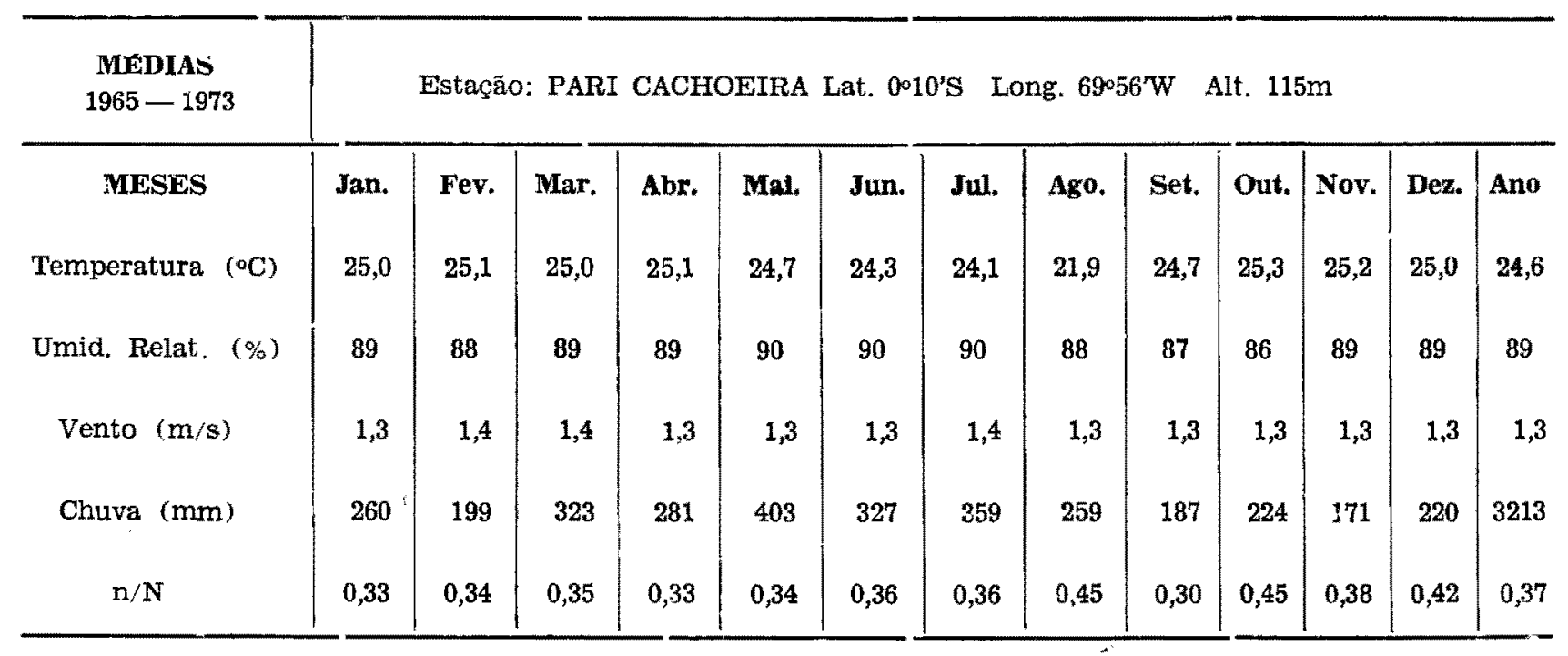




\begin{tabular}{|c|c|c|c|c|c|c|c|c|c|c|c|c|c|}
\hline $\begin{array}{c}\text { MÉDIAS } \\
1961-1973\end{array}$ & \multicolumn{5}{|c|}{ Estação: PARINTINS } & \multicolumn{2}{|c|}{ Lat. $2^{\circ} 38^{\prime}$ S } & \multicolumn{2}{|c|}{ Long. $56^{\circ} 44 \mathrm{~W}$} & \multicolumn{2}{|c|}{ Alt. $32 \mathrm{~m}$} & & \\
\hline MESES & Jan. & Fev. & Mar. & Abr. & Mai. & Jun. & Jul. & Ago. & Set. & Out. & Nov. & Dez. & Ano \\
\hline Temperatura $\left({ }^{\circ} \mathrm{C}\right)$ & 26,8 & 26,3 & 26,3 & 26,3 & 26,4 & 26,7 & 26,7 & 27,5 & 28,0 & 28,4 & 28,0 & 27,4 & 27,1 \\
\hline Umid. Relat. (\%) & 86 & 87 & 88 & 89 & 89 & 87 & 84 & 81 & 77 & 76 & 78 & 82 & 84 \\
\hline Vento $(\mathrm{m} / \mathrm{s})$ & 1,9 & 2,0 & 1,8 & 1,7 & 1,6 & 1,6 & 1,7 & 1,7 & 2,1 & 2,1 & 1,9 & 1,8 & 1,8 \\
\hline Chuva $(\mathrm{mm})$ & 250 & 279 & 324 & 356 & 346 & 200 & 112 & 88 & 41 & 77 & 142 . & 161 & 2376 \\
\hline $\mathrm{n} / \mathrm{N}$ & 0,40 & 0,37 & 0,34 & 0,38 & 0,43 & 0,57 & 0,67 & 0,72 & 0,63 & 0,69 & 0,56 & 0,53 & 0,52 \\
\hline
\end{tabular}

\begin{tabular}{|c|c|c|c|c|c|c|c|c|c|c|c|c|c|}
\hline $\begin{array}{c}\text { MÉDIAS } \\
1961-1973\end{array}$ & \multicolumn{5}{|c|}{ Estação: PORTO VELHO } & \multicolumn{2}{|c|}{ Lat. $8^{\circ} 46^{\prime} \mathrm{S}$} & \multicolumn{2}{|c|}{ Long. $63^{\circ} 55^{\top} \mathrm{W}$} & \multicolumn{2}{|c|}{ Alt. $105 \mathrm{~m}$} & & \\
\hline MESES & Jan. & Fev. & Mar. & Abr. & Mai & Jun. & Jul. & Ago. & Set. & Out. & Nov. & Dez. & Ano \\
\hline Temperatura $\left({ }^{\circ} \mathrm{C}\right)$ & 25,6 & 25,5 & 25,6 & 25,8 & 25,6 & 25,4 & 25,4 & 26,7 & 26,6 & 26,3 & 25,3 & 25,8 & 25,9 \\
\hline Umid. Relat. (\%) & 89 & 89 & 88 & 87 & 85 & 81 & 76 & 73 & 78 & 83 & 86 & 88 & 84 \\
\hline Vento $(\mathrm{m} / \mathrm{s})$ & 1,4 & 1,3 & 1,2 & 1,3 & 1,3 & 1,3 & 1,5 & 1,2 & 1,5 & 1,5 & 1,4 & 1,3 & 1,4 \\
\hline Chuva $(\mathrm{mm})$ & 265 & 307 & 283 & 254 & 134 & 39 & 27 & 42 & 111 & 186 & 222 & 288 & 2158 \\
\hline $\mathrm{n} / \mathrm{N}$ & 0,31 & 0,29 & 0,34 & 0,37 & 0,51 & 0,64 & 0,73 & 0,67 & 0,55 & 0,46 & 0,38 & 0,34 & 0,47 \\
\hline
\end{tabular}

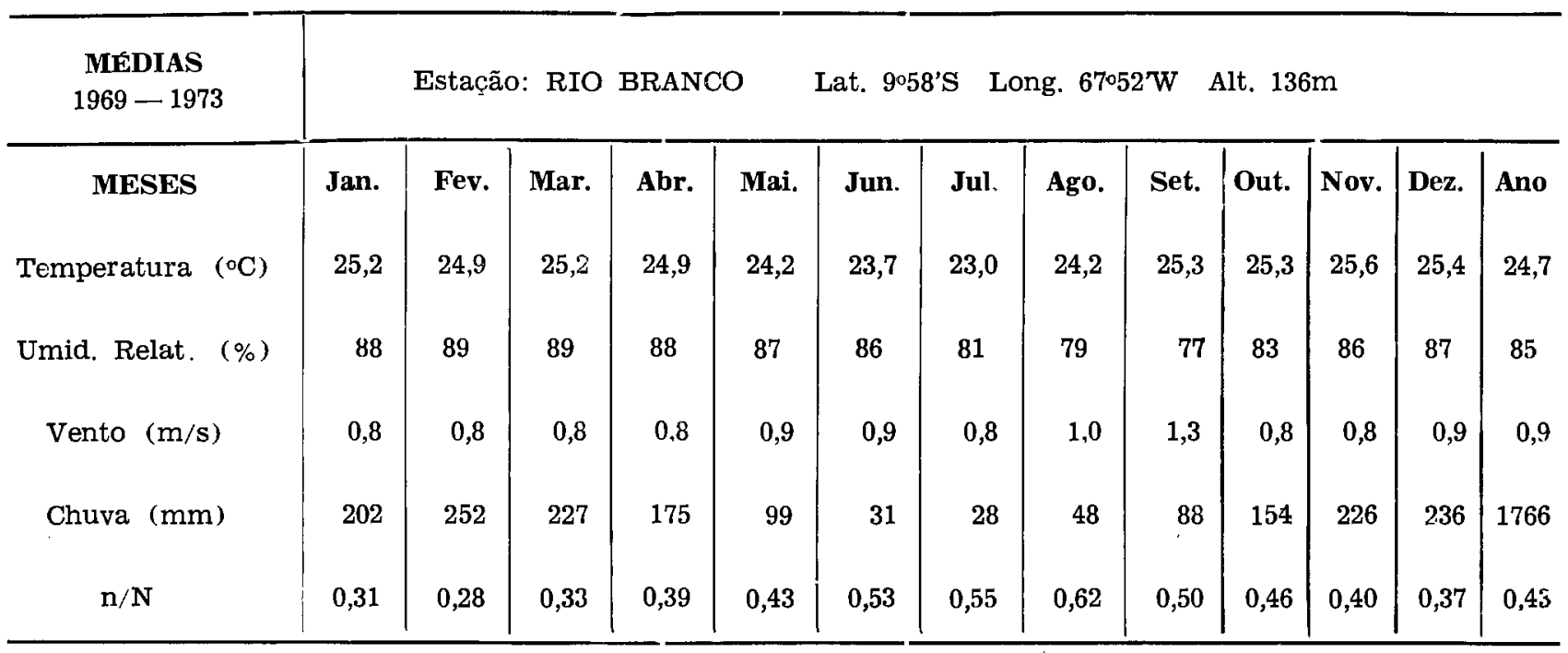




\begin{tabular}{|c|c|c|c|c|c|c|c|c|c|c|c|c|c|}
\hline \multirow{3}{*}{$\begin{array}{c}\text { MEDIAS } \\
1966-1973 \\
\text { MESES }\end{array}$} & \multicolumn{13}{|c|}{ SANTA ISABEL DO } \\
\hline & \multicolumn{5}{|c|}{ Estaģăo: RIO NEGRO } & \multicolumn{2}{|c|}{ Lat. 0020'S } & ng. 65 & 'W & Alt. 45 & & & \\
\hline & Jan. & Fev. & Mar. & Abr. & Mai. & Jun. & Jul. & Ago. & Set. & Out. & Nov. & Dez. & Ano \\
\hline Temperatura $\left({ }^{\circ} \mathrm{C}\right)$ & 25,5 & 25,7 & 25,7 & 25,5 & 25,3 & 25,1 & 24,8 & 25,1 & 25,6 & 26,1 & 26,2 & 26,0 & 25,6 \\
\hline Umid. Relat. (\%) & 89 & 89 & 90 & 90 & 91 & 91 & 90 & 90 & 88 & 87 & 87 & 88 & 89 \\
\hline Vento $(\mathrm{m} / \mathrm{s})$ & 1,6 & 1,8 & 1,8 & 1,6 & 1,6 & 1,6 & 1,6 & 1,7 & 1,7 & 1,8 & 1,7 & 1,7 & 1,7 \\
\hline Chuva $(\mathrm{mm})$ & 211 & 179 & 282 & 291 & 306 & 243 & 199 & 197 & 168 & 126 & 180 & 174 & 2556 \\
\hline $\mathrm{n} / \mathrm{N}$ & 0,44 & 0,41 & 0,36 & 0,35 & 0,33 & 0,38 & 0,42 & 0,46 & 0,48 & 0,50 & 0,43 & 0,41 & 0,41 \\
\hline
\end{tabular}

\begin{tabular}{|c|c|c|c|c|c|c|c|c|c|c|c|c|c|}
\hline $\begin{array}{c}\text { MEDIAS } \\
1931-1960\end{array}$ & \multicolumn{5}{|c|}{ Estação: SANTAREM } & \multicolumn{2}{|c|}{ Lat. $2^{\circ 25}$ 'S } & 1g. 54 & \multicolumn{3}{|c|}{$W$ Alt. $20 \mathrm{~m}$} & & \\
\hline MESES & Jan. & Fev. & Mar. & Abr. & Mai. & Jun. & Jul. & Ago. & Set. & Out. & Nov. & Dez. & Ano \\
\hline Temperatura $\left({ }^{\circ} \mathrm{C}\right)$ & 25,8 & 25,5 & 25,5 & 25,6 & 25,6 & 25,4 & 25,4 & 26,2 & 26,7 & 27,0 & 26,9 & 26,5 & 26,0 \\
\hline Umid. Relat. $(\%)$ & 85 & 87 & 88 & 88 & 89 & 88 & 36 & 83 & 80 & 78 & 79 & 80 & 84 \\
\hline Vento $(\mathrm{m} / \mathrm{s})$ & 2,0 & 1,7 & 1,7 & 1,6 & 1,5 & 1,7 & 1,8 & 2,4 & 2,8 & 3,1 & 2,8 & 2,4 & 2,1 \\
\hline Chuva (mm) & 179 & 275 & 358 & 362 & 293 & 174 & 112 & 50 & 39 & 46 & 85 & 123 & 2096 \\
\hline $\mathrm{n} / \mathrm{N}$ & 0,38 & 0,31 & 0,29 & 0,34 & 0,39 & 0,49 & 0,57 & 0,57 & 0,61 & 0,61 & 0,52 & 0,50 & 0,47 \\
\hline
\end{tabular}

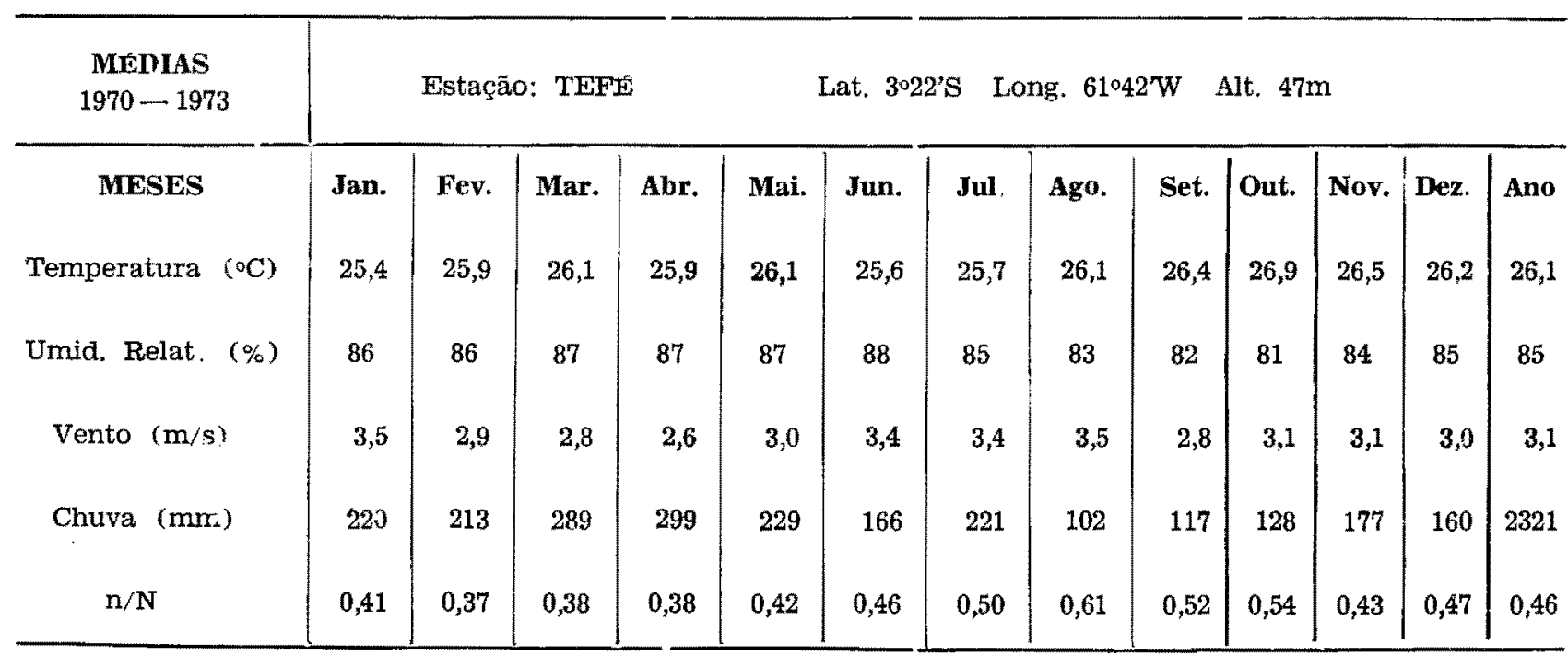


Tabela I - (continuação)

\begin{tabular}{|c|c|c|c|c|c|c|c|c|c|c|c|c|c|}
\hline $\begin{array}{c}\text { MÉdIAS } \\
1931-1960\end{array}$ & \multicolumn{5}{|c|}{ Estação: UAUPĒS } & at. $0^{\circ}$ & \multicolumn{3}{|c|}{ Long. $67 \circ 05 \mathrm{~W}$} & \multicolumn{4}{|c|}{ Alt. $85 \mathrm{~m}$} \\
\hline MESES & Jan. & Fev. & Mar. & Abr. & Mai. & Jun. & Jul. & Ago. & Set. & Out. & Nov. & Dez. & Ano \\
\hline Temperatura $\left({ }^{\circ} \mathrm{C}\right)$ & 25,4 & 25,5 & 25,6 & 25,3 & 25,0 & 24,5 & 24,3 & 24,8 & 25,4 & 25,6 & 25,9 & 25,5 & 25,2 \\
\hline Umid. Relat. (\%) & 89 & 87 & 87 & 89 & 90 & 91 & 90 & 88 & 86 & 86 & 87 & 88 & 88 \\
\hline Vento $(\mathrm{m} / \mathrm{s})$ & 0,5 & 0,6 & 0,5 & 0,4 & 0,4 & 0,5 & 0,4 & 0,4 & 0,5 & 0,6 & 0,6 & 0,5 & 0,5 \\
\hline Chuva $(\mathrm{mm})$ & 274 & 250 & 285 & 267 & 317 & 250 & 246 & 195 & 148 & 173 & 202 & 305 & 2912 \\
\hline $\mathrm{n} / \mathrm{N}$ & 0,43 & 0,46 & 0,43 & 0,40 & 0,41 & 0,41 & 0,44 & 0,50 & 0,55 & 0,51 & 0,50 & 0,46 & 0,46 \\
\hline
\end{tabular}

TABELA II - Valores do coeficiente médio mensal de ajuste (f) da evapotranspiração potencial estimada (E $_{T}$ ) em função da relação $E_{H} / E_{T}$ m $\mathbf{m}$ m

\begin{tabular}{llllllllllllllllll}
\hline CIDADES & COEFICIENTES. & Jan & Fev & Mar & Abr & Mai & Jun & Jul & Ago & Set & Out & Nov & Dez \\
\hline & $\mathbf{E}_{\mathbf{T}}$ & & & & & & & & & & & & \\
& 4,1 & 3,8 & 3,6 & 3,8 & 4,1 & 4,4 & 4,6 & 4,9 & 5,0 & 5,2 & 5,0 & 4,7
\end{tabular}

BELEM $\quad \mathbf{E}$

$\mathrm{Hi}$

$\begin{array}{llllllllllll}3,6 & 3,5 & 3,3 & 3,4 & 3,6 & 3,8 & 4,0 & 4,3 & 4,4 & 4,5 & 4,3 & 4,0\end{array}$

$\mathbf{f}=\mathrm{E}_{\mathrm{H}} / \mathrm{E}$

$\begin{array}{llllllllllll}0,88 & 0,92 & 0,92 & 0,89 & 0,88 & 0,86 & 0,87 & 0,88 & 0,88 & 0,87 & 0,86 & 0,85\end{array}$

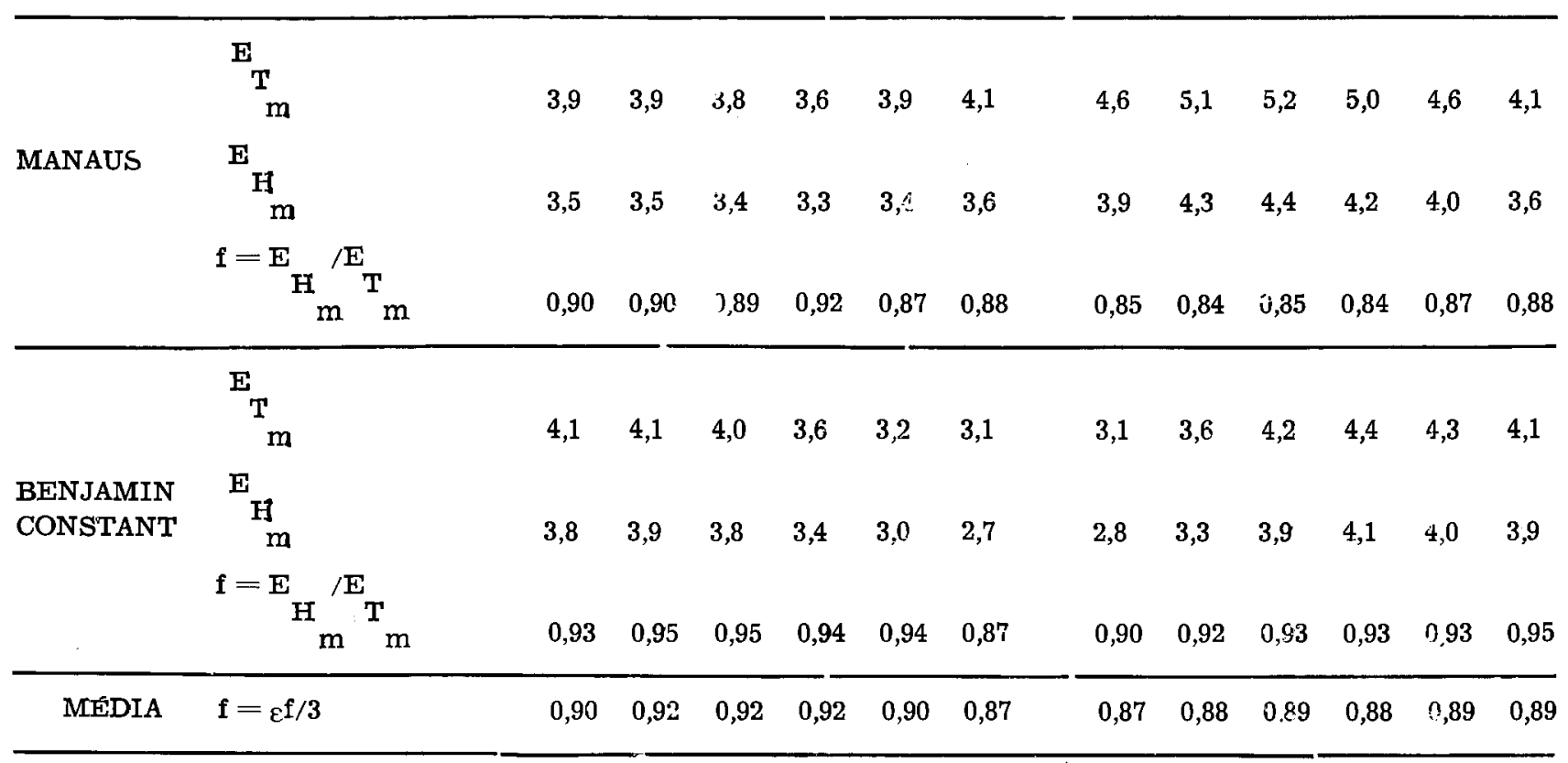

Estimativa da evapotranspiração... 
TABELA III - Valores médios de evapotranspiração potencỉal estimado pelo método de PENMAN mo. dificado.

Evapotranspiraçäo potencial estimada $(\underset{\mathbf{T}}{\mathbf{E}}) \mathrm{em} \mathrm{mm} / \mathrm{dia}$

$\mathbf{m}$

\begin{tabular}{|c|c|c|c|c|c|c|c|c|c|c|c|c|c|}
\hline CIDADES & Jan & Fev & Mar & $\mathbf{A b r}$ & Mai & Jun & Jul & Ago & Set & Out & Nov & Dez & Ano \\
\hline ALTAMIRA & 3,6 & 3,5 & 3,3 & 3,2 & 3,4 & 2,8 & 3,4 & 4,2 & 4,3 & 4,1 & 3,6 & 3,6 & 3,6 \\
\hline ALTO TAPAJÓS & 3,4 & 3,6 & 3,5 & 3,5 & 3,6 & 3,6 & 3,7 & 4,4 & 4,2 & 4,4 & 3,9 & 3,6 & 3,8 \\
\hline BARCELOS & 4,1 & 4,3 & 4,3 & 3,8 & 3,6 & 3,6 & 3,8 & 4,1 & 4,5 & 4,5 & 4,3 & 4.2 & 4,1 \\
\hline BENJAMIN CONSTANT & 4,1 & 4,2 & 4,0 & 3,6 & 3,3 & 3,1 & 3,1 & 3,6 & 4,2 & 4,4 & 4,3 & 4,1 & 3,8 \\
\hline CARAUARI & 4,2 & 3,8 & 3,8 & 3,3 & 3,2 & 3,4 & 3,5 & 3,9 & 4,2 & 4,2 & 3,7 & 3,5 & 3,7 \\
\hline COARI & 3,7 & 3,9 & 3,6 & 3,3 & 3,5 & 3,6 & 4,0 & 4,4 & 4,6 & 4,4 & 4,2 & 3.9 & 3,9 \\
\hline FONTE BOA & 4,0 & 4,1 & 4,0 & 3,8 & 3,5 & 3,4 & 3,4 & 3,8 & 4,3 & 4,3 & 4,2 & 4,1 & 3,9 \\
\hline HUMAITA & 4,0 & 3,8 & 4,9 & 3,6 & 3,4 & 3,5 & 3,7 & 4,3 & 4,6 & 4,6 & 4,3 & 4.3 & 4,1 \\
\hline YAUARETÊ & 3,7 & 3,7 & 3,7 & 3,6 & 3,2 & 3,1 & 3,2 & 3,6 & 4,0 & 3,9 & 3,8 & 3,6 & 3,6 \\
\hline MACAPA & 4,2 & 4,1 & 3,8 & 3,6 & 3,6 & 4,0 & 4,3 & 4,6 & 5,6 & 5,9 & 5,5 & 4,6 & 4,5 \\
\hline MANAUS & 3,9 & 3,9 & 3,8 & 3,6 & 3,9 & 4,2 & 4,6 & 5,1 & 5,2 & 5,0 & 4,6 & 4,1 & 4,3 \\
\hline PARI-CACHOEIRA & 3,5 & 3,7 & 3,8 & 3,6 & 3,3 & 3,2 & 3,3 & 3,6 & 3,5 & 4,1 & 3,7 & 3,7 & 3,6 \\
\hline PARINTINS & 4,1 & 4,0 & 3,9 & 4,0 & 3,7 & 4,1 & 4,5 & 5,1 & 5,2 & 5,5 & 5,0 & 4,5 & 4.5 \\
\hline PORTO VELHO & 3,8 & 3,8 & 3,8 & 3,6 & 3,6 & 3,7 & 4,0 & 4,5 & 4,6 & 4,4 & 4,2 & 3,9 & 4,0 \\
\hline RIO BRANCO & 3,8 & 3,7 & 3,8 & 3,5 & 3,1 & 3,1 & 3,2 & 3,9 & 4,2 & 4,3 & 4,2 & 4,1 & 3,7 \\
\hline SANTA ISABEL & 4,0 & 4,0 & 3,9 & 3,7 & 3,3 & 3,4 & 3,5 & 3,8 & 4,2 & 4,4 & 4,1 & 3,5 & 3,8 \\
\hline SANTAREM & 4,0 & 3,7 & 3,7 & 3,7 & 3,5 & 3,7 & 4,0 & 4,4 & 5,0 & 5,2 & 4,7 & 4,4 & 4,2 \\
\hline TEFE & 4,2 & 4,1 & 4,1 & 3,8 & 3,7 & 3,5 & 3,8 & 4,5 & 4,6 & 4,9 & 4,4 & 4,4 & 4,2 \\
\hline UAUPES & 4,0 & 4,2 & 4,1 & 3,8 & 3,5 & 3,4 & 3,5 & 3,9 & 4,4 & 4,3 & 4,2 & 3,9 & 3,9 \\
\hline & & & & & & & & & & & \multicolumn{3}{|c|}{ MÉDIA } \\
\hline
\end{tabular}




\section{ERRATA}

No Resumo (página 215) e no Summary (página 220):

\section{Onde lê:}

... através da precipitação, $14,4 \times 10^{12} \mathrm{~m}^{3}$ de água por ano, sendo este total ... e uma evapotranspiração de $8,9 \times 10^{12} \mathrm{~m}^{3}$ /ano. Desde que a transpiração pelas plantas representa $61,8 \%$ do balanço hídrico.

Leia-se:

... através da precipitação, $12,0 \times 10^{12} \mathrm{~m}^{3}$ de água por ano, sendo este total ... e uma evapotranspiração de $6,48 \times 10^{12} \mathrm{~m}^{3}$ /ano. Desde que a transpiração pelas plantas representa $54 \%$ da precipitação.

\section{Summary: Where it reads:}

... Amazon Basin receives $14.4 \times 10^{12} \mathrm{~m}^{3}$ water/year ... and an evapotranspiration of $8.9 \times 10^{12} \mathrm{~m}^{3} /$ year. Since transpiration by plants represents $61.8 \%$ of the water balance.

\section{Please read:}

... Amazon Basin receives $12.0 \times 10^{12} \mathrm{~m}^{3}$ water/year ... and an evapotranspiration of $6.48 \times 10^{12} \mathrm{~m}^{3} /$ year. Since transpiration by plants represents $54 \%$ of the precipitation. 